\title{
Tratamiento de Fracturas en Mandíbulas Atróficas: Presentación de Dos Casos Clínicos
}

\author{
Management of Atrophic Mandible Fractures: Report of Two Clinical Cases
}

\author{
Marcelo Mardones M."'; Eduardo Navia G."'; Rodrigo Bravo A."** \& Cristopher Mayer O."*
}

\begin{abstract}
MARDONES, M. M.; NAVIA, G. E.; BRAVO, A. R. \& MAYER, O. C. Tratamiento de fracturas en mandíbulas atróficas: Presentación de dos casos clínicos. Int. J. Odontostomat., 5(2):126-132, 2011.

RESUMEN: El siguiente trabajo tiene por objetivo presentar la experiencia del equipo de Cirugía Maxilofacial del Hospital San José, para el manejo de las fracturas en mandíbulas atróficas, reportando el tratamiento médico-quirúrgico y evolución post operatoria de casos clínicos. Se presentan dos casos clínicos de pacientes de sexo femenino, con enfermedades sistémicas de base, edéntulas, con diagnóstico de fractura en mandíbula atrófica consecutivos a caídas de nivel. De los casos tratados, el equipo logró resultados satisfactorios, mediante un tratamiento integral para los pacientes con este tipo de fracturas, basado en protocolos actuales encontrados en la literatura. El tratamiento de elección para este tipo de fracturas corresponde a la reducción anatómica quirúrgica y fijación mediante placas y tornilos de osteosíntesis. Fundamentado por sus favorables resultados y a la rápida rehabilitación funcional del paciente. El manejo multidisciplinario es fundamental para tratar este tipo de pacientes, por la posible concomitancia de enfermedades de base que pudiesen poseer estos pacientes.
\end{abstract}

PALABRAS CLAVE: fractura mandibular, fracturas en mandíbulas atróficas, tratamiento fractura mandibular atrófica.

\section{INTRODUCCIÓN}

Las fracturas de mandíbulas edéntulas atróficas es una patología importante para los cirujanos debido a las dificultades asociadas a la pérdida severa de tejido óseo y a la pobre calidad y vascularización de este tejido. La atrofia mandibular conduce a una disminución de la masa ósea, provocando que el hueso sea más frágil y vulnerable a las fracturas. La atrofia es la etapa final de los pacientes edéntulos, comenzando este proceso desde que una pieza dental se pierde, desencadenando una serie de efectos biológicos que conducen a la pérdida de la apófisis alveolar (Ellis \& Price, 2008), esta pérdida de masa ósea y la disminución de la vascularización, provocan una disminución de la resistencia mandibular lo que la hace más vulnerable a las fracturas (Marciani, 2001).

Usualmente estas fracturas ocurren en pacientes adultos mayores, con capacidad osteogénica reducida por una disminución de sus células osteoprogenitoras y morfológicamente por la presen- cia mayoritaria de hueso cortical, acompañado de insuficiente irrigación sanguínea, características asociadas a la atrofia ósea mandibular (Aziz \& Najjar, 2009). Luhr desarrolló una clasificación basada en el grado de atrofia ósea, el cual es de utilidad para el tratamiento de este tipo de mandíbulas, determinando: Grado I (altura ósea de $16-20 \mathrm{~mm}$ ), Grado II (altura ósea de $11-15 \mathrm{~mm}$ ) y Grado III (altura ósea menor de $10 \mathrm{~mm}$ ) (Wittwer et al., 2006).

Este tipo de fracturas son poco comunes comparadas con las fracturas mandibulares en pacientes dentados, por lo tanto la mayoría de los cirujanos tiene experiencia limitada en el tratamiento de este tipo de fracturas (Madsen \& Haug, 2006). Mugino trató 335 fracturas mandibulares en un periodo de 14 años (1980 a 2004) en el que sólo un $2 \%$ eran fracturas de mandíbulas atróficas (Aziz \& Najjar), porcentaje que concuerda con otros estudios (Madsen \& Haug). La distribución según la región anatómica comprometida por este

\footnotetext{
*Cirujano Maxilofacial. Complejo Hospitalario San José, Santiago de Chile, Chile.

* Docente del Departamento de Cirugía y Traumatología Oral y Maxilofacial, Facultad Odontología, Universidad de Chile, Chile.

${ }^{* * *}$ Residente de Cirugía Maxilofacial Complejo Hospitalario San José, Santiago de Chile, Chile.
} 
tipo de fracturas tiende a ser diferente a la de los pacientes dentados, con un alto porcentaje de fracturas en la región del cuerpo mandibular $(43,5 \%)$ y bajos porcentajes en el ángulo $(15,2 \%)$ y sínfisis mandibular (4,3\%) (Miloro, 2004).

Es posible observar complicaciones asociadas al tratamiento de esta patología, provocadas por el estado sistémico general de este tipo de pacientes y complicaciones biomecánicas por la pérdida de masa ósea, generando dificultades en la estabilización y fijación de los segmentos (Ellis \& Price). Condiciones que reportan una alteración en la consolidación ósea en un $20 \%$ de los casos (Wittwer et al.), pudiendo observarse una pseudoartrosis o hipoestesia del nervio alveolar inferior (Ellis \& Price).

El tratamiento quirúrgico para las fracturas en pacientes dentados y desdentados, tienen los mismos principios básicos, los cuales son reducción, fijación y estabilización, para poder lograr restaurar la morfología y función mandibular. Los métodos de tratamiento no quirúrgicos mediante inmovilización a través de fijaciones máxilo mandibulares, en este tipo de pacientes, son complejas de realizar por la falta de piezas dentarias y la reducida sección transversal de la mandíbula (Thaller \& McDonald, 2004).

La combinación de factores biomecánicos y biológicos desfavorables, como la reducida sección transversal mandibular, la mínima área de contacto entre los rasgos de fractura, escazo periostio y endostio para nutrir las células ósteo progenitoras que generan un hueso denso y esclerótico (Fonseca, 2004), pueden determinar resultados desfavorables en el tratamiento de esta patología. Estos factores determinan que sea recomendable tratar las fracturas en mandíbulas atróficas de manera más invasiva, mediante un acceso amplio, una reducción quirúrgica anatómica y fijación con un elemento de osteosíntesis de mayor rigidez (Barber, 2001), determinando resultados más favorables (Wittwer et al.).

Un número no despreciable de estos pacientes, son médicamente comprometidos, pudiendo presentar enfermedades de base como diabetes, hipertensión y/o enfermedad de las arterias coronarias, osteoporosis, lo que determina un manejo pre, intra y post operatorio complejo (Aziz \& Najjar). Bajo estas condiciones, las opciones de tratamiento deben ser cuidadosamente analizadas, ya que el estado sistémico del paciente podría determinar la contraindicación de un procedimiento quirúrgico bajo anestesia general, justificando un manejo de la patología mediante un tratamiento no quirúrgico con la necesidad de observación, cuidados y controles periódicos, aunque el tratamiento de elección sea el quirúrgico.

Los riesgos pre-operatorios, en este tipo de fracturas y en estos pacientes, han llevado a algunos cirujanos a considerar como tratamiento ideal la reducción cerrada o no quirúrgica (Barber).

\section{REPORTE DE CASOS}

Se presentan dos casos clínicos de pacientes de género femenino, de la tercera edad, tratados el año 2009, que ingresaron al Servicio de Cirugía Máxilo Facial del Hospital San José con diagnóstico de fractura en mandíbula atrófica consecutivas a caídas de nivel. El primer paciente tenía una fractura de cuerpo y ángulo derecho en la mandíbula edéntula con grado de atrofia ósea de tipo II. El segundo paciente tenía una fractura de cuerpo bilateral mandibular con similar grado de atrofía mandibular que el primer paciente. Ambos pacientes presentaban antecedentes sistémicos como hipertensión arterial y una de ellas sumaba una patología psiquiátrica, ambas estables y controladas farmacológicamente.

El diagnóstico se obtuvo a través del examen clínico e imagenológico (Fig. 1) mediante una tomografía computada (TC), donde se determinan los rasgos de fracturas y la ubicación de elementos anatómicos a considerar al momento de la reconstrucción, como el canal alveolar mandibular. Se solicitaron los pases médicos correspondientes para un procedimiento quirúrgico bajo anestesia general.

El procedimiento quirúrgico consistió en un acceso al plano óseo mandibular a través de un abordaje submandibular extraoral (Fig. 2) hasta la identificacion del rasgo de fractura (Fig. 3), se realizó la reducción anatómica de los cabos fracturarios para posteriormente realizar la fijación mediante una placa de 2,4mm y tornillos de osteosíntesis bicorticales (Fig. 4), para finalmente realizar el cierre por planos del abordaje quirúrgico. El paciente permanece en cuidados intermedios por 24 horas post operatorias para asegurar una correcta recuperación anestésica-quirúrgica, luego permanece 48 horas hospitalizado en habitación médico-quirúrgica, donde se solicita una TC de control y se da el alta hospitalaria con citación a controles ambulatorios (Fig. 5). 

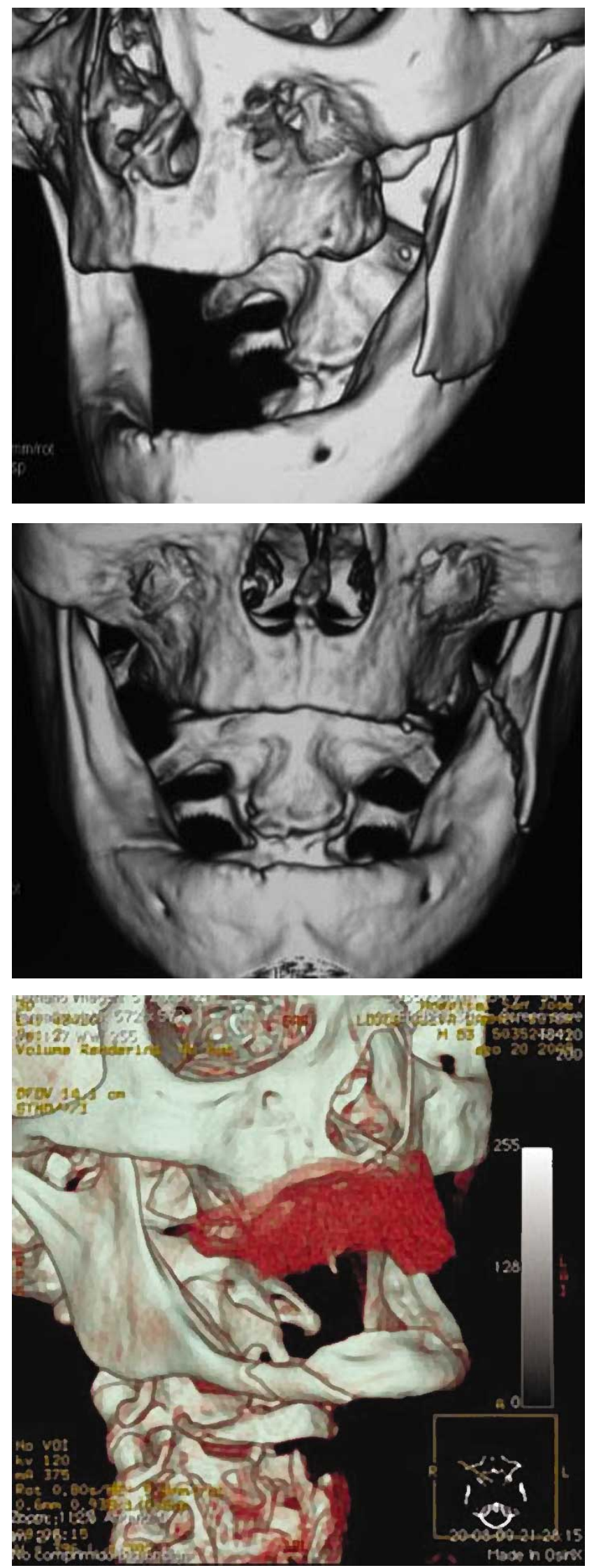

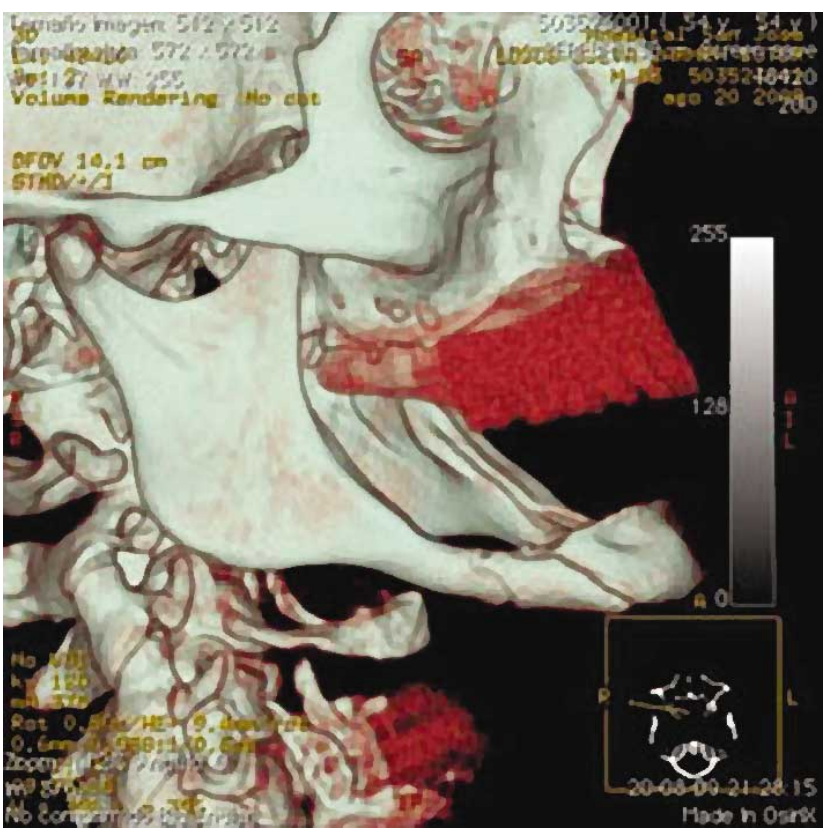

Fig. 1. Reconstrucción 3D pre-operatorio.

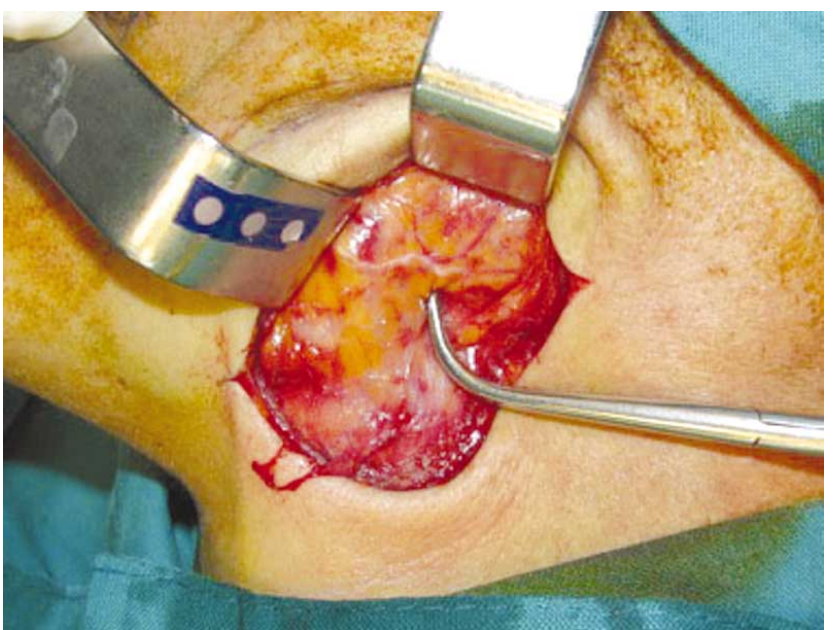

Fig. 2. Abordaje submandibular (Extraoral).

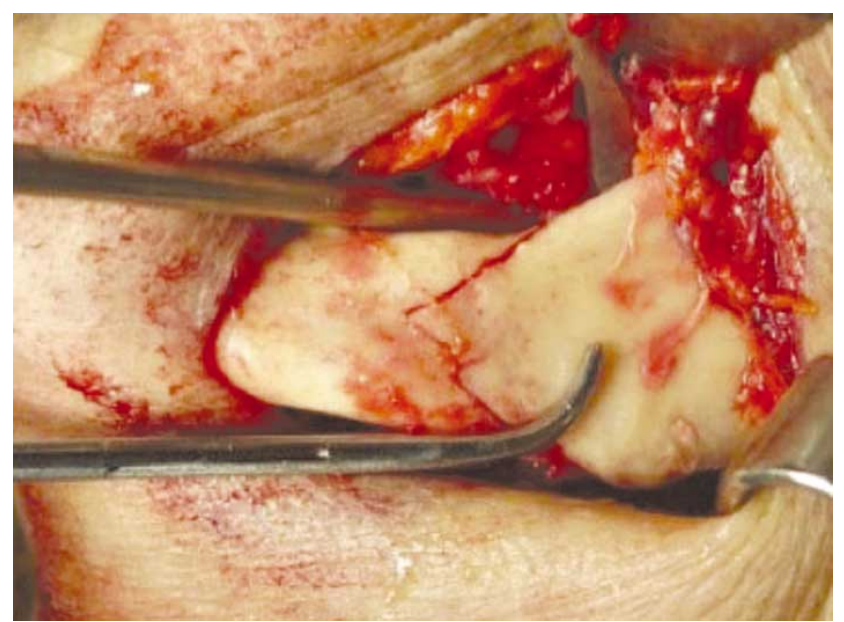

Fig. 3. Identificación y reducción de rasgo de fractura. 

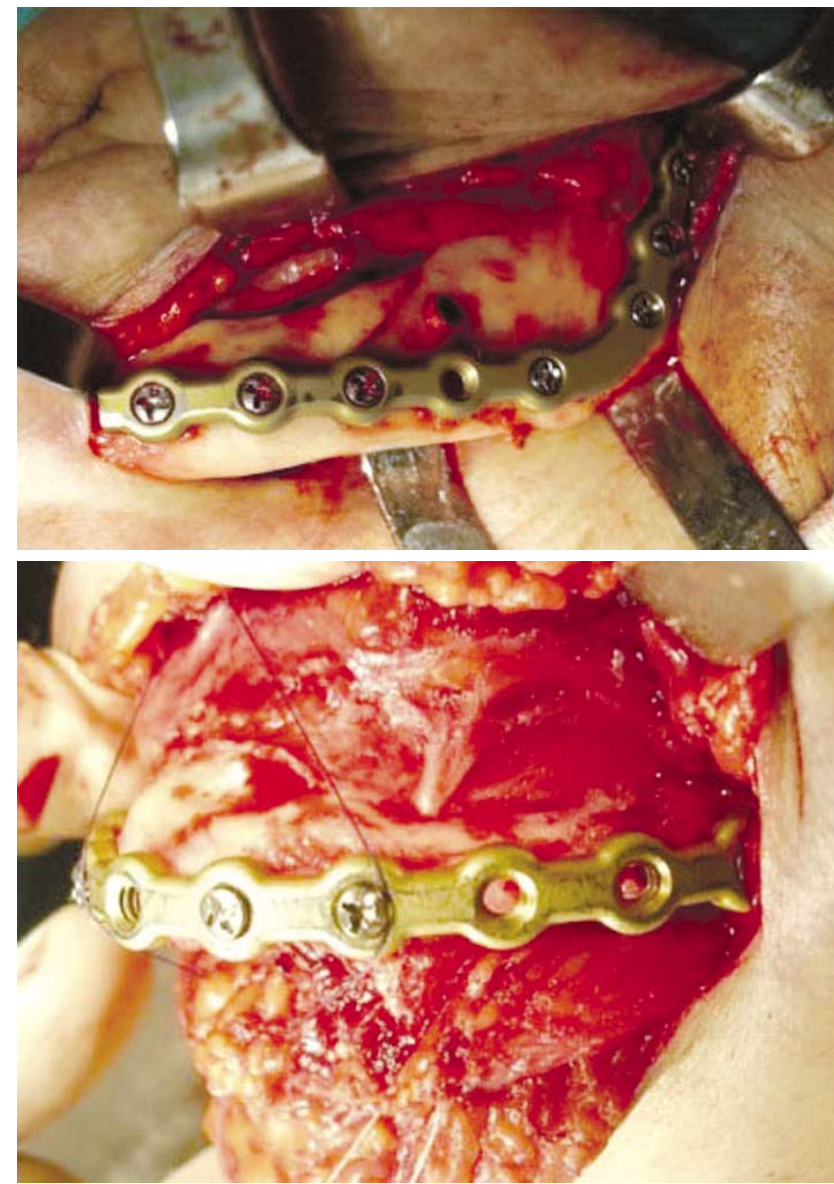

Fig. 4. Reducción y fijación anatómica con placas y tornillos de osteosíntesis.

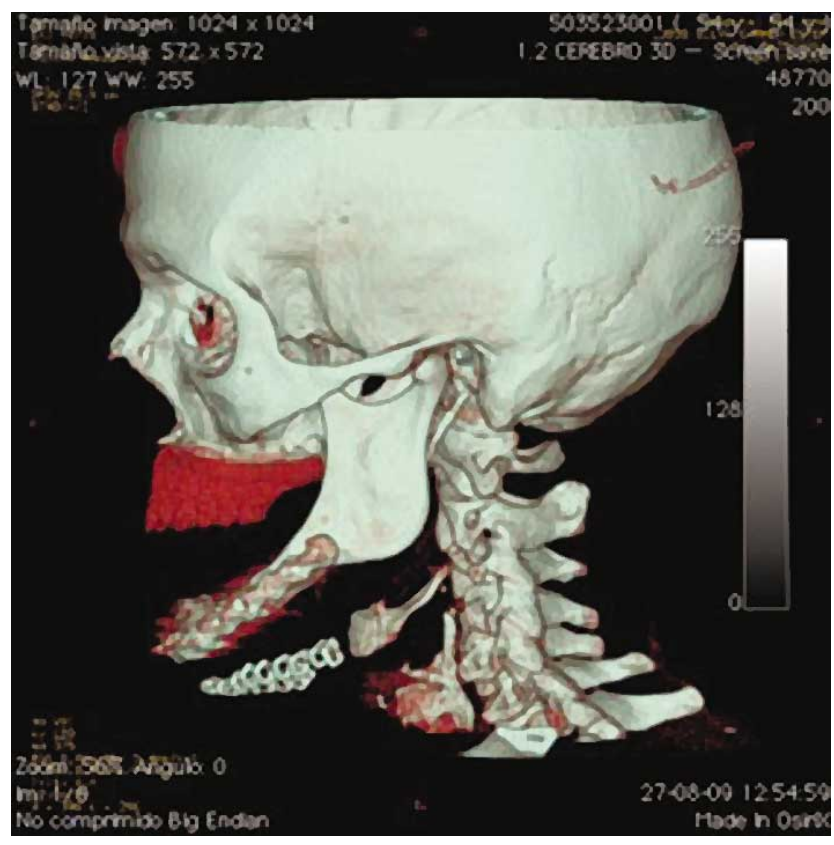

Fig. 5. Reconstrucción 3D de control post-operatorio.
En el post operatorio se presentaron condiciones esperables como edema, dolor leve a moderado y equimosis (Fig. 6), aunque el resultado inmediato del tratamiento fué muy favorable con una rápida rehabilitación funcional del paciente, sin secuelas neurológicas, tanto sensitivas como motoras (Fig. 7). Al sexto día de control no se observaron signos de equimosis extra e intraoral (Fig. 8). El acceso extraoral tuvo una cicatrización adecuada sin secuelas cosméticas ni funcionales (Fig. 9).

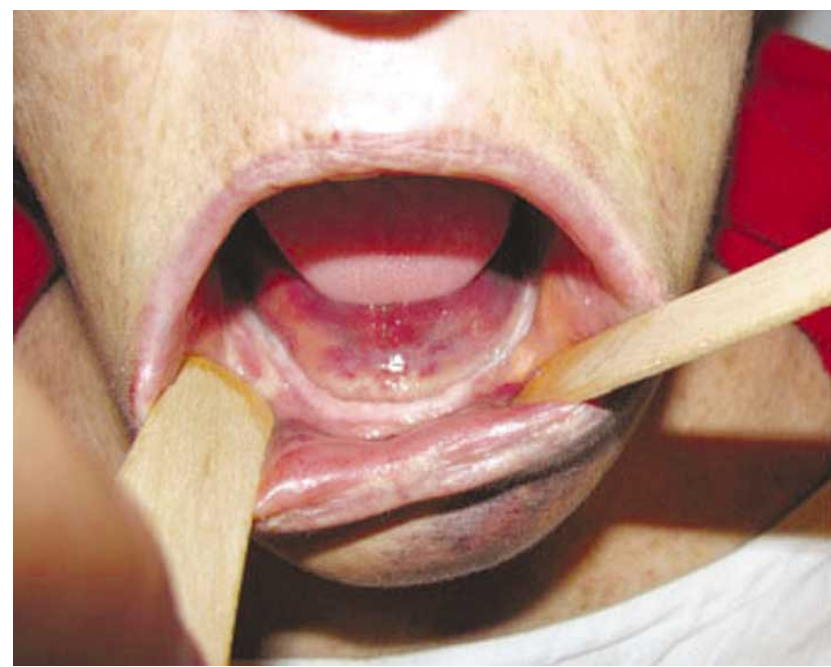

Fig. 6. Control tercer día post-operatorio. Presencia de equímosis intraoral y extraoral.

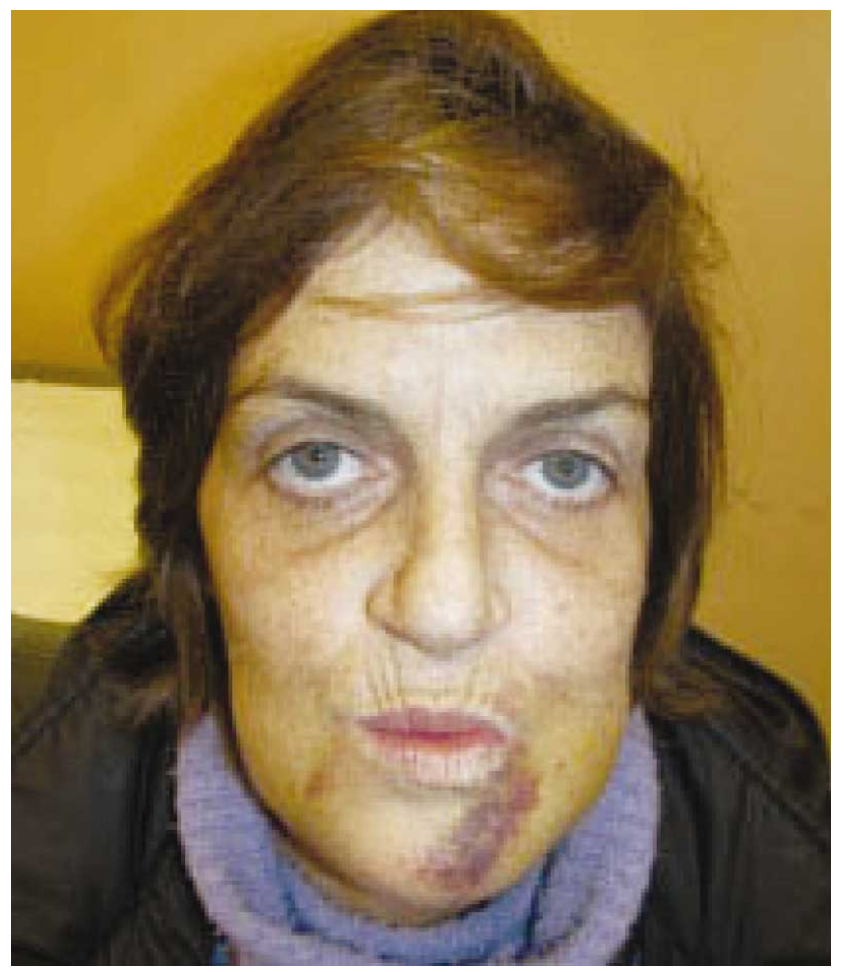

Fig. 7. Control sexto día post-operatorio. Indemnidad ramo curvo nervio facial. 


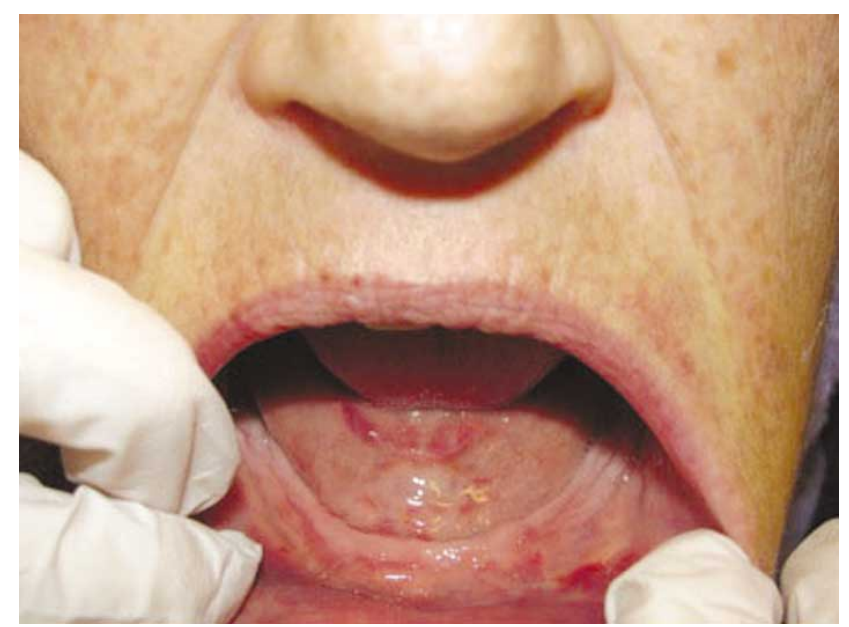

Fig. 8. Control Sexto día post-operatorio.

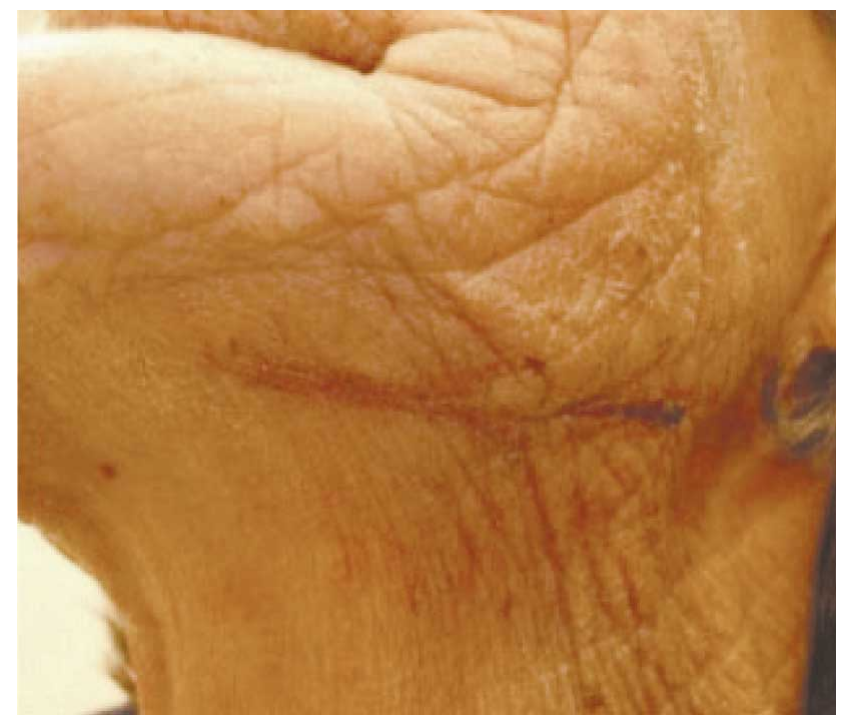

Fig. 9. Cicatriz acceso extraoral.

\section{DISCUSIÓN}

Existen controversias con respecto al tipo de tratamiento para las fracturas de mandíbulas atróficas (Wittwer et al.), algunos autores proponen que la mayoría de las fracturas deberían ser tratadas de manera no quirúrgica con prótesis y bloqueo intermaxilar (Thaller \& McDonald), ya que Bradley describió mediante angiografía que la arteria alveolar inferior no es un suministro consistente en mandíbulas atróficas y que probablemente el suministro principal es por el plexo sanguíneo del periostio, el cual durante el procedimiento quirúrgico es disecado poniendo en riesgo el suministro sanguíneo del hueso mandibular, provocando una alteración en la consolidación ósea (Madsen \& Haug).
Por otra parte, otros autores proponen que éstas fracturas pueden ser tratadas satisfactoriamente mediante un tratamiento quirúrgico. Estudios realizados en los últimos 15 años, han demostrado que la reducción quirúrgica no incrementa la alteración en la consolidación de la fractura en las mandíbulas atróficas (Barber). J. Lyons Academy Study revisaron 167 fracturas en 104 mandíbulas edéntulas atróficas, de los pacientes tratados quirúrgicamente, el 15\% tuvo como complicación una pseudoartrósis, en cambio los que fueron tratados de manera no-quirúrgica el $20 \%$ a $26 \%$ tuvo la misma complicación (Thaller \& McDonald).

En pacientes donde las fracturas mandibulares atróficas tienen como características mínimo o nulo desplazamiento, sin exposición ósea intraoral o con uniones fibrosas, tratadas mediante tratamiento no quirúrgico, pueden tener un calidad de vida razonable, más aún si no utilizan prótesis dentales (Madsen \& Haug). En cambio, para los pacientes que médicamente pueden someterse a un procedimiento quirúrgico bajo anestesia general, la reducción abierta y fijación interna con placas de osteosíntesis es la técnica de tratamiento de elección. Las ventajas de este tratamiento son la visualización directa de la fractura, reducción anatómica, estabilidad de los fragmentos y el temprano retorno a la función normal. A diferencia del tratamiento no quirúrgico que no siempre logra una adecuada reducción anatómica de los fragmentos y se caracteriza por un mayor tiempo de evolución y duración del tratamiento, provocando incomodidad en el paciente y con una rehabilitación funcional más tardía (Ellis \& Price).

En 1996 Luhr et al. demostraron que la reducción abierta tiene mejores resultados que el tratamiento no quirúrgico; en la actualidad existe un consenso que la reducción quirúrgica con fijación interna es el tratamiento de elección.

Con respecto al abordaje quirúrgico también es un tema controversial, ya que algunos autores proponen el acceso intraoral y otros el extraoral. Según estudios se ha demostrado que no existe evidencia que demuestre la superioridad de uno versus el otro (Wittwer et al.), por lo tanto el uso depende de la preferencia y competencia de cada cirujano (Ellis \& Price).

La ventaja del acceso intraoral es la menor complejidad en realizarlo, ausencia de cicatriz externa, menor riesgo de injuria al nervio facial y cierre de un solo plano anatómico (Ellis \& Price). La desventaja es la presencia del nervio mentoniano, el cual se encuen- 
tra presente en la parte superior del hueso mandibular, el cual puede ser dañado en el momento de la incisión y disección, provocando hipostesia hemilabial. Esta complicación depende del grado de atrofia del hueso mandibular que puede exponer este nervio a una porción más cercana al reborde alveolar (Wittwer et al.); otro riesgo es la posible contaminación de la fractura por la flora bacteriana oral, aumentando el riesgo de infección intra y post operatoria. Otra desventaja sería la dificultad intrínseca del acceso para lograr una adecuada visualización, reducción y fijación de la fractura (Ellis \& Price).

El acceso extraoral tiene como desventajas dejar una cicatriz no deseable en el paciente, provocar una posible injuria al nervio facial y a otras estructuras anatómicas, situaciones que pueden minimizarse con un operador entrenado y con la adecuada expertiz. Tanto la reducción y fijación interna de la fractura mediante un abordaje extraoral se ve facilitada por la visión directa que otorga éste, disminuyendo la complejidad del procedimiento en relación a si se realiza de manera intraoral. Factores que determinan que sea el acceso de elección para los casos presentados (Aziz \& Najjar).

La dimensión de las placas de osteosíntesis para el tratamiento también es foco de diversos estudios, donde en los últimos 30 años ha existido debate acerca del tamaño y número de placas de osteosíntesis requeridas para este tipo de fracturas (Ellis \& Price), en la literatura, la dimensiones varían desde placas 0,5 a 3,5 mm. Thaller y otros clínicos proponen utilizar las placas más pequeñas posibles y de bajo perfil para obtener los mejores resultados (Latrou et al., 1998) y para evitar daño al nervio dentario inferior con los tornillos. Bruce, Ellis y Mugino prefieren placas de la mayor rigidez posible, para dar una mejor biomecánica y estabilidad mandibular para que las fuerzas sean resistidas por la placa y favorecer una adecuada cicatrización ósea (Ellis \& Price, 2008). Por otra parte, Marciani \& Hill proponen el uso de mallas de titanio con injerto óseo de cresta iliaca inmediata (Wittwer et al.). Sikes et al. (2000) recomiendan el uso de placas de reconstrucción $2,4 \mathrm{~mm}$.

En general el sistema de osteosíntesis que se propone utilizar en este tipo de fracturas, son placas de la mayor rigidez posible, ya que análisis biomecánicos de pacientes desdentados, demostraron que a nivel del cuerpo mandibular se reciben fuerzas de distintas direcciones durante la función mandibular, con rotación superior de la rama mandibular producida por los músculos masticadores y simultáneamente los músculos suprahioideos provocan una rotación inferior produciendo un movimiento de torsión, por lo que debiesen ocuparse placas que resistan esta biomecánica y aliviar en parte de éstas fuerzas al tejido óseo para favorecer la cicatrización (Tucker, 2000).

La A. O. (Arbeitsgemeinschaft fur Osteosynthesefrage) recomienda sistemas de placas de reconstrucción, que abarquen la zona de las fracturas y fijación en las zonas de tejido óseo sano y estable, proponiendo el uso de placas de reconstrucción $2.4 \mathrm{~mm}$ (Ellis \& Madsen) indicando una regla: "hueso mandibular débil, la placa de osteosíntesis mas rígida debe ser utilizada" (Prein, 1998).

Esta última propuesta adoptada por nosotros, coincide con nuestro criterio para la elección de los elementos de osteosíntesis para el tratamiento de este tipo de fracturas.

El uso de tornillos bicorticales o mono corticales dependerá del grado de atrofia ósea mandibular, ya que si la fractura se produce en una mandíbula severamente atrófica probablemente el remanente óseo no permitirá instalar tornillos que perforen ambas tablas óseas por el inminente daño al nervio alveolar inferior que se podría producir, en tal caso debiese optarse por tornillos monocorticales y un estricto control postoperatorio. Los casos presentados permitieron el uso de tornillos bicorticales. Esta situación es fácilmente evaluable de forma previa mediante el estudio imagenológico (TAC).

Importante es mencionar que los casos clínicos pueden variar según la condición sistémica y ósea del paciente, pudiendo existir múltiples factores que ayuden al éxito del tratamiento, así consideramos que un elemento importante en la planificación pre-quirúrgica, es la confección de un modelo estereolitográfico pre-quirúrgico de la mandíbula traumatizada. Examen complementario que en nuestra realidad asistencial hospitalaria no nos permite realizar, sin embargo, las ventajas de contar con un modelo estereolitográfico pre-quirúrgico serían realizar un estudio más acabado del caso, elegir y adaptar los elementos de osteosíntesis a ocupar en forma previa, con el fin de disminuir el tiempo operatorio quirúrgico, que en este tipo de pacientes es importante considerar.

En conclusión, el manejo de las fracturas en mandíbulas atróficas es diverso, pudiendo ser quirúr- 
gicos como no quirúrgicos. Este debe ser elegido basado en el tipo de fractura, su desplazamiento, grado de atrofia y experiencia del equipo tratante, considerando además los factores sistémicos individuales de cada paciente. El tratamiento de elección para las fracturas en mandibulas atróficas, según la experiencia del equipo de Cirugía Máxilo Facial del Hospital San José, corresponde a la reducción quirúrgica mediante acceso extraoral y fijación a través de placas y tornillos de osteosíntesis, debido a sus favorables resultados y a la rápida rehabilitación funcional del paciente. El manejo multidisciplinario es fundamental para tratar este tipo de pacientes, por la concomitancia de enfermedades de base que pudiesen poseer estos pacientes.

MARDONES, M. M.; NAVIA, G. E.; BRAVO, A. R. \& MAYER, O. C. Management of atrophic mandible fractures: Report of two clinical cases. Int. J. Odontostomat., 5(2):1126-132, 2011.

ABSTRACT: The objective of the present case report is to present the experience of the maxillofacial surgery team of the Hospital San Jose in the management of atrophic mandible fracture, through the medical-surgical treatment and postoperatory evolution of two clinical cases. We studied two women with systemic conditions, edentulous with a diagnosis of atrophic mandible fracture subsequent to fall injury. In all treated cases the surgical team achieved satisfactory results through an integral treatment of patients with this type of fracture, based on actual literature reported protocols. The treatment of choice for this type of fractures is an anatomical open reduction, by internal fixation with osteosynthesis plates and screws on the basis of favorable results and functional rehabilitation of patients. The multidisciplinary management is fundamental for treatment of this type of patient due to the concomitant systemic conditions of these patients.

\section{KEY WORDS: mandible fracture, atrofic mandible fractures, atrofic mandible fracture treatment.}

\section{REFERENCIAS BIBLIOGRÁFICAS}

Aziz, S. R. \& Najjar, T. Management of the edentulous/atrophic mandibular fracture. Atlas Oral Maxillofac. Surg. Clin. North Am., 17(1):75-9, 2009.

Barber, H. D. Conservative management of the fractured atrophic edentulous mandible. J. Oral Maxillofac. Surg., 59(7):789-91, 2001.

Ellis, E. 3rd. \& Price, C. Treatment protocol for fractures of the atrophic mandible. J. Oral Maxillofac. Surg., 66(3):421-35, 2008.

Fonseca, R. Oral and Maxillofacial Trauma. Philadelphia, W. B. Saunders, 2004. pp.473-526. V. 1.

latrou, I.; Samaras, C. \& Theologie-Lygidakis, N. Miniplate osteosynthesis for fractures of the edentulous mandible: a clinical study 1989-96. J. Craniomaxillofac. Surg., 26(6):400-4, 1998.

Luhr, H. G.; Reidick, T. \& Merten, H. A. Results of treatment of fractures of the atrophic edentulous mandible by compression plating: a retrospective evaluation of 84 consecutive cases. J. Oral Maxillofac. Surg., 54(3):250-5, 1996.

Madsen, M. J. \& Haug, R. H. A biomechanical comparison of 2 techniques for reconstructing atrophic edentulous mandible fractures. J. Oral Maxillofac. Surg., 64(3):457-65, 2006.

Marciani, R. D. Invasive management of the fractured atrophic edentulous mandible. J. Oral Maxillofac. Surg., 59(7):7925, 2001.
Miloro, M. Peterson's principles of oral and maxillofacial surgery. $2^{\text {nd }}$ ed. London, BC Decker, 2004. pp.401-33.

Prein, J. Manual of Internal Fixation in the Cranio-Facial Skeleton. Berlin, Springer, 1998. pp.57-92.

Sikes, J. W. Jr.; Smith, B. R. \& Mukherjee, D. P. An in vitro study of the effect of bony buttressing on fixation strength of a fractured atrophic edentulous mandible model. J. Oral Maxillofac. Surg., 58(1):56-62, 2000.

Thaller, S. R. \& McDonald, W. S. Facial trauma. New York, Marcel Dekker, 2004. pp.381-416.

Tucker, M. R. An in vitro study of the effect of bony buttressing on fixation strength of a fractured atrophic edentulous mandible model. J. Oral Maxillofac. Surg., 58(1):62, 2000.

Wittwer, G.; Adeyemo, W. L.; Turhani, D. \& Ploder, O. Treatment of atrophic mandibular fractures based on the degree of atrophy--experience with different plating systems: a retrospective study. J. Oral Maxillofac. Surg., 64(2):230-4, 2006.

Dirección para correspondencia:

Marcelo Mardones Muñoz

Calle Sergio Livingstone Pohlhammer 943

Independencia, Santiago

CHILE

Mail:drmardones@gmail.com

Recibido : 13-06-2011

Aceptado: 20-07-2011 